\title{
MODELAGEM E SIMULAÇÃO NUMÉRICA DO TRANSPORTE REATIVO DE ESPÉCIES QUÍMICAS EM MEIOS POROSOS
}

\author{
N. F. MEDEIROS ${ }^{1}$, S.A.F. SILVA ${ }^{1}$, F. F. VIEIRA ${ }^{1}$ \\ ${ }^{1}$ Universidade Estadual da Paraíba, Departamento de Engenharia Sanitária e Ambiental \\ E-mail para contato: Nayrlon@ hotmail.com
}

\begin{abstract}
RESUMO - A preocupação com os recursos naturais tem sido crescente visto que sua disponibilidade é um fator limitante para o desenvolvimento socioeconômico. Nesse contexto o presente trabalho retrata a contaminação do solo por derivados de petróleo devido a ocorrência de vazamentos em tanques combustíveis em virtude de seu mal estado de conservação. O estudo objetiva o desenvolvimento de um modelo matemático e simulação numérica do transporte reativo de espécies químicas para entendimento de como um determinado poluente se comporta ao ser transportado no solo considerando parâmetros como coeficiente de difusão, reação química, retardo e velocidade de escoamento através da análise e previsão de cenários. O problema é composto de meio poroso com apenas uma camada e considera-se a transferência de massa bidirecional transiente e Reação química irreversível, utilizou-se a técnica da transformada integral generalizada. Pode-se visualizar a influência dos parâmetros, onde os mesmos prevêem se a pluma do poluente desloca-se para regiões mais profundas ou próximas da superfície.
\end{abstract}

\section{INTRODUÇÃO}

Agentes como demasiado crescimento demográfico, urbanização acelerada e desenvolvimento industrial tem contribuído direta e indiretamente na qualidade das águas. A preocupação com esse recurso natural tem sido crescente principalmente nas últimas décadas visto que sua disponibilidade é um fator limitante para o desenvolvimento socioeconômico. Segundo as agências de proteção ambiental, o derramamento de poluentes no solo e nas águas devido a vazamentos ou acidentes de derivados de petróleo é crescente, havendo sempre a preocupação de como evitar tais ocorrências e como remediar as que já aconteceram. Corseuil e Martins (1997) dizem que destes, a gasolina merece destaque, seja pela quantidade envolvida, ou pela periculosidade, verificada pela liberação, ao entrar em contanto com a água subterrânea, dos compostos BTEX (benzeno, tolueno, etil-benzeno e xilenos), que são substâncias depressoras do sistema nervoso central e causadoras de leucemia.

Nessa perspectiva, a modelagem matemática pode ser inserida como ferramenta para tomada de decisão, pois ela possibilita a geração de cenários futuros, podendo-se assim ter um controle da poluição gerada. Segundo Azevedo et al. (1996) os avanços computacionais aliados aos métodos numéricos, têm permitido o desenvolvimento de modelos que descrevem o transporte de solutos no solo, cuja vantagem principal do uso de modelos é a economia de tempo e capital investido, haja vista que possibilitam simular múltiplos cenários, ao invés de se utilizar longos períodos de observação e de coleta de dados. 
O presente trabalho objetiva o desenvolvimento de um modelo matemático e simulação numérica do transporte reativo de espécies químicas em meios porosos, ou seja, faz um estudo que possibilita o entendimento de como um determinado poluente se comporta ao ser transportado no solo considerando parâmetros como coeficiente de difusão, velocidade de escoamento, coeficiente de reação química e o coeficiente de retardo.

\section{REVISÃO TEÓRICA}

\subsection{Poluição do solo e a modelagem matemática como ferramenta para sistemas ambientais}

Para Costa e Teixeira (2010, apud Fleck et al., 2013) o enfoque preventivo do controle ambiental se tornou necessário para determinar os efeitos da ação humana sobre a integridade química, física e biológica do meio natural. Dentro deste contexto, surgem os modelos matemáticos de qualidade da água, como importantes instrumentos de controle da poluição hídrica. A modelagem é uma ferramenta muito importante que pode ser usada em sistemas ambientais, seus objetivos são interpretar e analisar o comportamento de um sistema, otimizar ou propor modificações, testar hipóteses e fazer precisões da resposta em diferentes condições operacionais. Pode-se dizer que o modelo matemático é uma simplificação da realidade, ou seja, ele aborda os parâmetros mais importantes a serem estudados.

Para Smith e Smith (2007) modelar é aplicar matemática a solução de problemas, e transformar um problema prático em um modelo matemático, buscando solução que possa ser aplicada na situação original. O objetivo de todo modelo é explicar o funcionamento de um determinado sistema. Nesse sentido a modelagem matemática pode simular cenários futuros, sendo essa uma das suas principais vantagens. Bastos (2010) diz que mesmo com as inúmeras aplicações, a modelagem matemática de ecossistemas vem sendo inserida aos poucos se tornando mais reconhecida, já que a necessidade crescente de respostas imediatas diante de problemas globais exige uma ferramenta holística, que seja hábil para o trabalho com fenômenos ambientais complexos.

Leal e Ruperti (2000) desenvolveram uma solução híbrida para um modelo bidimensional de seleção de transporte em um aquífero homogêneo com escoamento subterrâneo uniforme. A Técnica da Transformada Integral Generalizada (GITT) foi utilizada para prover a solução híbrida numéricoanalítica, e dois procedimentos numéricos foram utilizados, tendo sido selecionado um caso teste para verificar a eficácia das soluções propostas.

\section{METODOLOGIA}

O problema foi composto de meio poroso com apenas uma camada e considerou-se o problema de transferência de massa bidirecional transiente, com reação química irreversível, sendo constantes as propriedades dentro da camada. Assume-se a concentração injetada em $\mathrm{x}=0$ e fluxo do poluente, sendo funções conhecidas e dependentes do tempo. 


\subsection{Equação da continuidade}

O ponto de partida para o desenvolvimento das equações diferenciais que descrevem o transporte de solutos em materiais porosos foi considerar o fluxo de soluto que entra e sai em um dado elemento de volume fixado em relação ao domínio do escoamento.

De acordo com a conservação das massas pode-se realizar o balanço material para a espécie química, por meio da aplicação de volume de controle:

$\left[\begin{array}{l}\text { Fluxo mássico } \\ \text { entrando no } \\ \text { volume }\end{array}\right]-\left[\begin{array}{l}\text { Fluxo mássico } \\ \text { saindo do } \\ \text { volume }\end{array}\right] \pm\left[\begin{array}{l}\text { Perda ou Ganho } \\ \text { de Massa devido } \\ \text { a reações }\end{array}\right]=\left[\begin{array}{l}\text { Taxa de variação } \\ \text { de massa no } \\ \text { volume }\end{array}\right]$

A concentração do soluto $\mathrm{C}$ é definida como sendo a massa de soluto por unidade de volume de solução. A massa de soluto por unidade de volume em um meio poroso é então $\varepsilon C$. Para um meio homogêneo a porosidade é uma constante, portanto:

$\frac{\partial(s C)}{\partial x}=\frac{s \partial C}{\partial x}$

\subsection{Transporte de poluentes em meios porosos com reações químicas}

Para descrever o nosso problema utilizou-se as equações a seguir: sendo que W é a velocidade, D é o coeficiente de difusão e K é o coeficiente de reação química.

$$
\begin{aligned}
& \frac{\partial \mathrm{C}(\mathrm{x}, \mathrm{t})}{\partial \mathrm{t}}-\mathrm{W} \frac{\partial \mathrm{C}(\mathrm{x}, \mathrm{t})}{\partial \mathrm{x}}=\mathrm{D} \frac{\partial^{\mathrm{x}} \mathrm{C}(\mathrm{x}, \mathrm{t})}{\partial \mathrm{x}^{2}}-K \mathrm{C}(\mathrm{x}, \mathrm{t}) \\
& \mathrm{C}(0, \mathrm{t})=1.0 \\
& \mathrm{C}(\mathrm{x}, 0)=\mathrm{C}_{\alpha} \\
& \frac{\partial \mathrm{C}(\mathrm{x}, \mathrm{t})}{\partial \mathrm{x}}=0
\end{aligned}
$$

Para resolver a equação 3 utilizou-se a Técnica da Transformada Integral Generalizada (GITT) que é uma técnica criada a pouco mais de duas décadas, onde se incluem a unificação de soluções para diferentes classes de problemas relacionados a difusão e convecção.

\section{RESULTADOS E DISCUSSÃO}

\subsection{Análise do efeito da variação do coeficiente de difusão (D)}

Esta análise procurou compreender a influência da variação do coeficiente de difusão (D). Esse coeficiente é um parâmetro físico da equação de transporte de solutos no solo e expressa dois fenômenos de transporte aditivos: a difusão, que ocorre em razão da existência de gradiente de 
concentração, e a dispersão mecânica, que é um movimento proporcionado por variações na velocidade de deslocamento da solução dentro dos poros individuais e entre poros de diferentes formas, tamanhos e direções.

Avaliou-se os perfis de concentração conforme a figura 1 com a influência do coeficiente de difusão para os valores de (D) iguais 0,001 ; 0,5 e 1,5. E mantiveram-se os valores de coeficiente de retardo (R), Reação química (K) e velocidade (W), constantes e iguais a 1,00; 5,0 e 0,001.

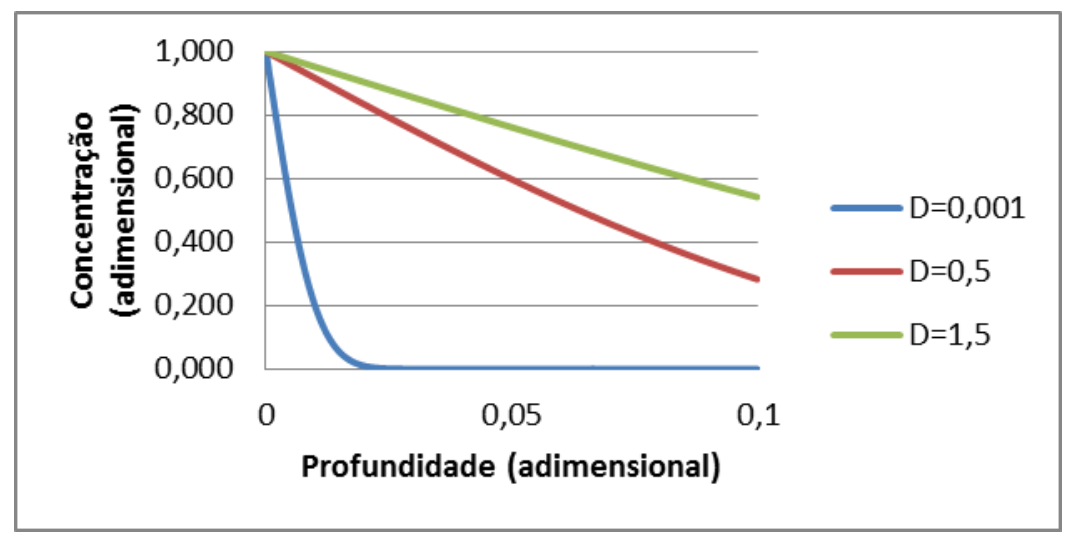

Figura 1: Perfil da concentração em função da profundidade, variando D e mantendo constante

$$
\mathrm{W}=0,001 \mathrm{R}=1,0 \text { e } \mathrm{K}=5,0 \text {. }
$$

Verificou-se que quanto maior o coeficiente de difusão maior é a propagação que a espécie química pode atingir no solo. Também vale salientar que independentemente da existência ou não de reações químicas, para elevados valores do coeficiente de difusão, a pluma de poluição atingirá regiões profundas do solo.

\subsection{Análise do efeito da variação do coeficiente de reação química (K)}

Nesta análise procurou-se compreender a influência do coeficiente de reação química (K). Esse coeficiente é responsável pela degradação do poluente no solo, onde sua presença ou ausência pode aumentar ou diminuir a chance, que o poluente possui para atingir grandes profundidades. Na Figura 2 estão ilustrados os perfis de concentração para valores de coeficiente de reação química $(\mathrm{K})$ iguais a 0,$0 ; 5,0$ e 30,0 . 


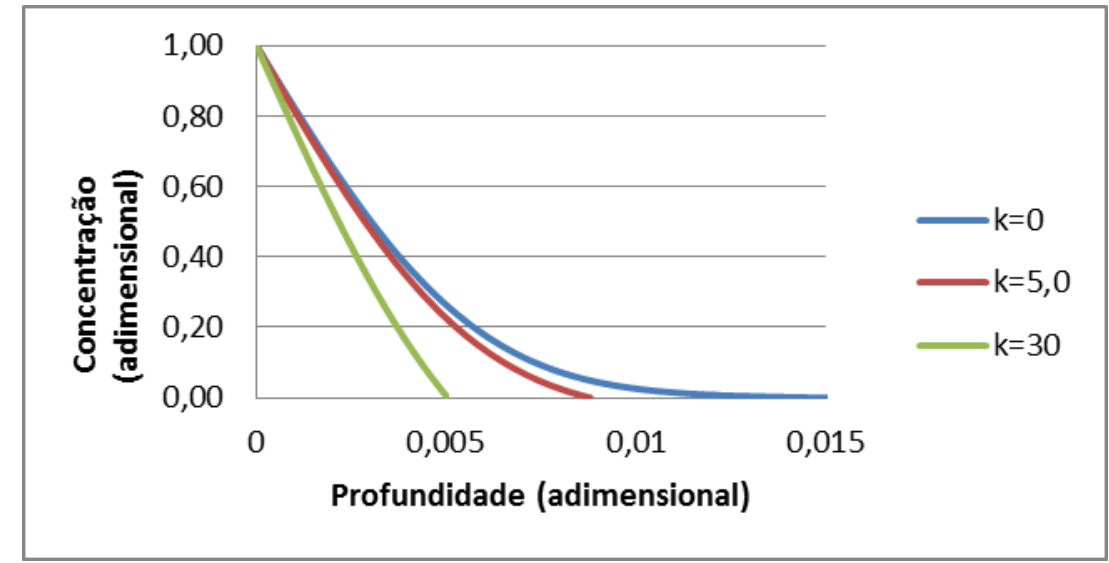

Figura 2: Perfil da concentração em função da profundidade, variando K, e mantendo constante

$$
\mathrm{W}=0,001 ; \mathrm{R}=1,0 \text { e } \mathrm{D}=0,001 \text {. }
$$

Observou-se que quanto maior o coeficiente de reação química menor é a profundidade que um determinado poluente atinge o solo, esse parâmetro é de grande importância, pois é um dos parâmetros capazes de informar se um determinado tipo de solo é capaz de se autorremediar.

\subsection{Análise do efeito da variação do coeficiente de retardo $(R)$ e da velocidade $(W)$}

O coeficiente de retardo nos fornece informação da dificuldade que a espécie poluente possui para se deslocar no meio poroso, ou seja, a capacidade que o meio possui para retardar o escoamento do poluente. Já a velocidade nos fornece informação da distancia em função do tempo que a espécie química é transportada.

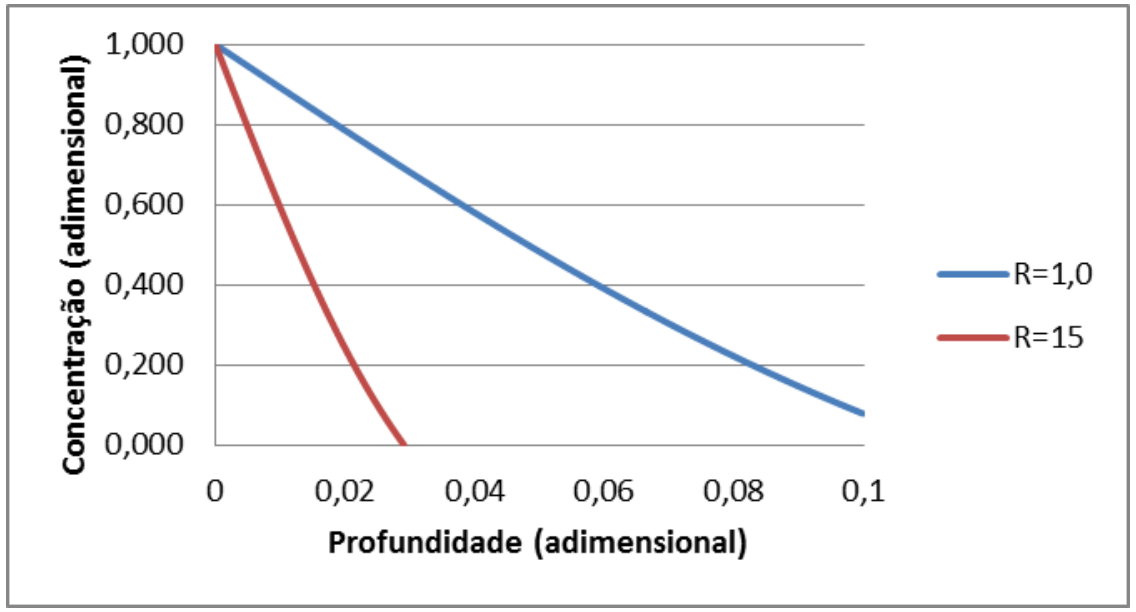

Figura 3: Perfil da concentração em função da profundidade, variando $R$, e mantendo constante $\mathrm{D}=0,5$ $\mathrm{W}=0,001 \mathrm{e} \mathrm{K}=30,0$. 
Observou-se na Figura 3 que quanto menor o coeficiente de retardo a pluma de poluição se desloca para regiões mais profundas do solo. E que para altos valores de coeficiente de retardo e de reação química o poluente é degradado mais rapidamente.

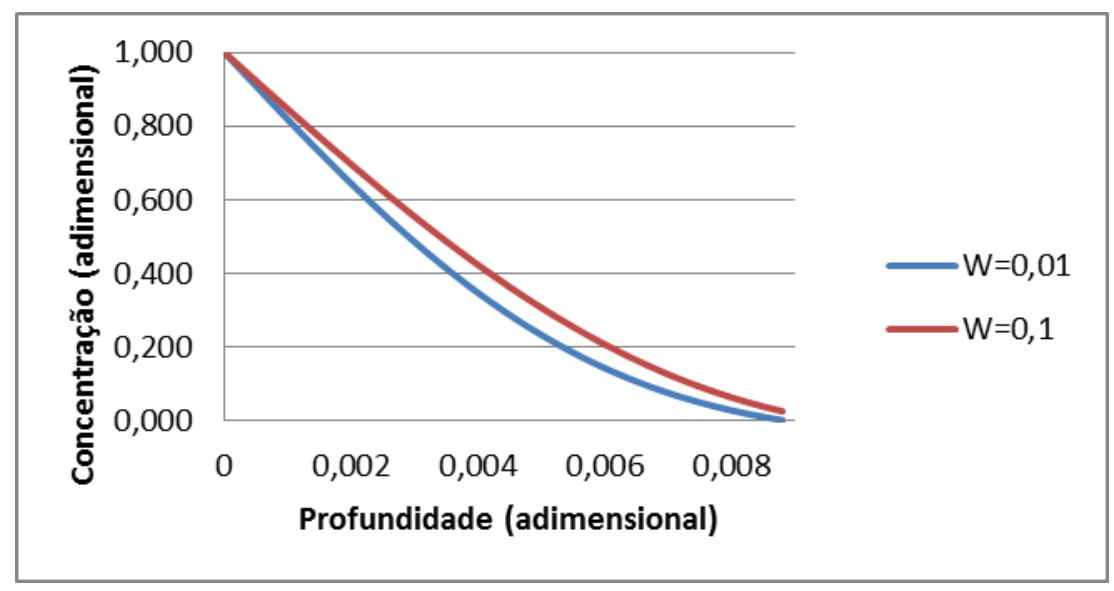

Figura 4: Perfil da concentração em função da velocidade, mantendo constante $\mathrm{D}=0,001 ; \mathrm{R}=1,0$ e $\mathrm{K}=5,0$.

Já a influência da velocidade retratada pela Figura 4 é menor se comparada ao comportamento da variação dos outros parâmetros estabelecidos nesse estudo.

\section{CONCLUSÃO}

O modelo desenvolvido para simular o comportamento dos poluentes é válido, mesmo com suas simplificações, visualizou-se que a influência dos parâmetros dos coeficientes de difusão, reação química e retardo e a velocidade de escoamento no solo. Tais parâmetros indicaram se a pluma do poluente irá se deslocar para regiões mais profundas ou se dispersar em regiões mais próximos da superfície.

Percebeu-se que o coeficiente de difusão assume um dos mais importantes fatores visto que se ele for alto, o determinado poluente irá se dispersar para regiões mais profundas da superfície. Já a velocidade de escoamento, apesar de importante, tem uma menor influência se comparada aos outros coeficientes com seus valores altos, uma vez que o coeficiente de retardo impede a passagem da espécie poluente no meio poroso e o coeficiente de reação química degrada o poluente presente.

A importância desse estudo se dá ao fato que é mais viável economicamente fazer uma simulação de como está sendo deslocada uma determinada espécie química poluente, para que se possa posteriormente fazer o planejamento e uma correta remediação da área, pois assim pode-se evitar maiores danos ao ambiente e ter um maior controle sobre os processos envolvidos. Dessa maneira a modelagem serve como uma ferramenta de prevenção e ajuda na tomada de decisão tanto na remediação de áreas poluídas, quanto para diversas atividades que tenham potencial poluidor. 


\section{REFERÊNCIAS}

AZEVEDO, A. S.; Kanwar, R. S.; Singh, P.; Pereira, L. S. Movement of $\mathrm{NO}_{3}$ - and atrazine through soil columns as affected by lime application. Transactions of the Asae, St. Joseph, v.39, n.3, p.937945, 1996.

BASTOS, Argemiro Midonês. Modelagem de Escoamento Ambiental como Subsídio à Gestão de Ecossistemas Aquáticos no Baixo Igarapé da Fortaleza - AP. 2010. 118 f. Dissertação (Mestrado) Curso de Pós-graduação em Biodiversidade Tropical, Universidade Federal do Amapá - Unifap, Macapá-ap, 2010

CORSEUIL, H. X., MARTINS, M. D. M., 1997, "Contaminação de Águas Subterrâneas por Derramamentos de Gasolina: O Problema é Grave?” In: Engenharia Sanitária e Ambiental, Vol. 2, No. 2, Abril/Junho, 1997.

COSTA, D. J. L.; TEIXEIRA, D. Aplicação de modelo de autodepuração para avaliação da qualidade da água do Ribeirão do Ouro, Araraquara-SP. Revista Uniara, n.1, v.13, p.49-62, 2010.

FLECK, Leandro; TAVARES, Maria Herminia Ferreira; EYNG, Eduardo. Principais modelos matemáticos de qualidade da água e suas aplicações: uma revisão. Revista Eletrônica Científica Inovação e Tecnologia, v. 1, n. 7, p.47-62, 2013.

LEAL, M. A.; RUPERTI, N. J. A Numerical Study for the Two-Dimensional Solute Transport in Groundwater Pathway via Integral Transform Method, Hybrid Methods in Engineering, New York, v. 2, n.1. 2000.

SMITH, J.; SMITH, P. How to develop a model. Published in 2007, by Oxford University Press. $125 \mathrm{p}$. 